\title{
Postmastectomy Radiation Treatment Rates as a Quality Measure: An Opportunity for Compliance Through Collaboration
}

\author{
Oluwadamilola M. Fayanju, MD ${ }^{1}$, Thomas A. Buchholz, $\mathrm{MD}^{2,3}$, and Kelly K. Hunt, MD ${ }^{1,4}$ \\ ${ }^{1}$ Department of Breast Surgical Oncology, The University of Texas MD Anderson Cancer Center, Houston, TX; \\ ${ }^{2}$ Department of Radiation Oncology, The University of Texas MD Anderson Cancer Center, Houston, TX; ${ }^{3}$ Office of the \\ Executive Vice President (EVP) and Physician-in-Chief, The University of Texas MD Anderson Cancer Center, Houston, \\ TX; ${ }^{4}$ Department of Surgical Oncology, The University of Texas MD Anderson Cancer Center, Houston, TX
}

During the past century, management of breast cancer has evolved from the Halstedian era approach of extensive surgical resection as the only hope for cure to a multidisciplinary treatment approach with locoregional and systemic therapies based on patient- and tumor-related factors. Multimodal therapy is often needed to establish both systemic and local control, and this approach has resulted in improved survival rates. Radiation therapy has come to play a crucial role in the setting of breast-conservation therapy and for selected patients treated with mastectomy. Adjuvant radiation therapy, routinely offered to patients with breast cancer after partial mastectomy, has been proven to decrease the risk of recurrence and improve breast-cancer-specific mortality compared with surgery alone. $^{1}$

Receipt of radiation therapy after breast-conserving surgery is high, with a recent survey demonstrating that $95 \%$ of women with strong indications for postoperative radiation went on to receive it. ${ }^{2}$ However, the same survey also demonstrated that only $77 \%$ of women with strong indications for postmastectomy radiation (PMRT) ultimately received it. This observed gap between recommended PMRT and actual rates of PMRT represents an important opportunity for clinical investigation and quality improvement. However, rectifying this problem will require not only additional research but also the

(C) Society of Surgical Oncology 2016

First Received: 10 March 2016;

Published Online: 9 May 2016

K. K. Hunt, MD

e-mail: khunt@mdanderson.org development of standardized quality measures generated by health care providers that can be readily measured and subsequently incorporated into practice.

Quality indicators, when widely adopted by members of a professional society or reported by an accrediting organization, have been shown to improve both provider performance and patient outcomes through a mechanism of bidirectional feedback. An excellent example of such synergy is provided by experience in the field of colorectal surgery. Reports from several studies ultimately led to a consensus that resection of at least 12 lymph nodes during colorectal surgery not only represents a sufficient number of nodes to be evaluated for accurate staging but also serves as a surrogate measure of high-quality surgical technique.

In 2008, according to a review of the National Cancer Data Base (NCDB), more than $60 \%$ of hospitals failed to meet the aforementioned quality benchmark. ${ }^{3}$ Resection of 12 or more nodes in $75 \%$ or more patients has since been adopted as an institutional quality metric by the American Society of Clinical Oncology (ASCO), the National Comprehensive Cancer Network (NCCN), the National Quality Forum (NQF), and the Commission on Cancer $(\mathrm{CoC})$. Compliance with this quality measure has gradually increased both in the United States and internationally, and some evidence indicates that patients at compliant hospitals have better long-term outcomes. ${ }^{4-6}$ However, some competing evidence, demonstrated in a secondary analysis of the Clinical Outcomes of Surgical Therapy (COST) trial comparing laparoscopic to open colectomy, shows that standardization and credentialing at both the institutional and individual levels may be more important than traditional surrogates of surgical quality in predicting patient outcomes after surgery. ${ }^{7}$ 
With regard to improving the quality of care for breast cancer, it is hard to fix what you can't measure. Thus, the extensive review of the NCDB by Minami et al. ${ }^{8}$ represents an important contribution to the literature. In their article, National Evaluation of the New Commission on Cancer Quality Measure for Post-Mastectomy Radiation, the authors report the results of their retrospective review of NCDB data abstracted for more than 750,000 mastectomies performed for invasive, nonmetastatic breast cancer from 1998 to 2011. They examined rates of PMRT in this cohort stratified by the period during which a patient's diagnosis occurred, clinicopathologic data (e.g., number of lymph nodes with cancer), and socioeconomic information (e.g., income level and distance from radiotherapy facility) to quantify changes in rates of PMRT receipt over time and to identify factors that might influence the decision to omit PMRT. The authors also sought to present their findings within the context of recently published guidelines, namely, the CoC 2014 endorsement of a new quality measure stating that adjuvant radiation should be recommended or administered for all breast cancer patients treated with mastectomy who have four or more positive lymph nodes. ${ }^{9}$

In addition, the $2016 \mathrm{NCCN}$ breast cancer guidelines "recommend" PMRT for patients with four or more involved lymph nodes, urge practitioners to "strongly consider" PMRT for patients with one to three positive nodes, and ask providers to "consider" PMRT for selected patients with node-negative disease (i.e., those with tumors $\geq 5 \mathrm{~cm}$ or with tumors $<5 \mathrm{~cm}$ with $<1$-mm margins). ${ }^{10}$ The authors found that by 2011, about two thirds of patients with four or more positive nodes received PMRT versus only $56 \%$ in 1998, with similar increases seen among patients with fewer than four positive nodes or even negative nodes. They found that PMRT was more likely to be omitted in older (i.e., age $\geq 70$ years) patients than in younger patients, independent of nodal status. Patients who lived more than 50 miles from a radiation treatment facility were less likely to receive PMRT than those who lived closer, again regardless of nodal status. In all nodal groups, patients from the lowest income quartile had lower rates of PMRT use than those from the highest income quartile. Importantly, patients at community cancer programs (those that diagnose $>100$ but $<500$ new cancers per year) were less likely to receive PMRT than patients at comprehensive community cancer programs (those with $>500$ new cancers diagnosed annually). ${ }^{11}$ Community cancer programs did not differ from academic/research cancer centers regarding the likelihood that PMRT would be used. The authors did not find a consistent relationship between race/ ethnicity and the likelihood of patients receiving PMRT.

The NCDB represents a collaboration between the CoC of the American College of Surgeons and the American
Cancer Society and is estimated to contain data on 70 $80 \%$ of all newly diagnosed cancers in the United States. Thus, the results of this study can reasonably be assumed to represent the extent to which PMRT is used throughout the United States. Administration of PMRT has increased over time, but there remains significant heterogeneity in its application.

The introduction of biologic subtypes of breast cancer may have been influential in treatment decisions during the period of this study, and the absence of this information is an important limitation of the data presented, as use of anatomic staging information alone can result in over- and undertreatment of patients. Another limitation of this study was that we did not know how often PMRT was recommended but not used in the care plans for patients in the study cohort. Among those with negative nodes in the current study, it is unclear whether the increase in PMRT use, from 8.3 to $10 \%$ from 1998 to 2011, represents an appropriate increase in utilization or possible overutilization among individuals for whom it might not be indicated. The lower rate of PMRT receipt among patients living more than 50 miles from the nearest radiation treatment facility is not surprising but remains regrettable in a health care environment that in other respects is becoming ever more patient-centered. Efforts should be devoted to redressing this disparity as well as that observed among those with lower incomes. These deficits highlight the important role that hypofractionated radiation regimens could play and the extent to which additional research into the long-term efficacy and safety of this approach should continue to be pursued.

Breast cancer represents a disease in which physicians from different specialties regularly collaborate to provide multidisciplinary, patient-centered care. Thus, the effort to increase appropriate use of PMRT should be approached in a similarly collaborative spirit. The decision to offer PMRT should be made with the input of not only surgical, radiation, and medical oncologists but also other team members such as plastic surgeons.

A unified opinion considering all patient- and tumorrelated factors will not only help manage patients' expectations but also reinforce providers' treatment recommendations. A recent study demonstrated that patients who underwent reconstruction were less likely to receive PMRT, even if indicated by their final pathologic stage after neoadjuvant chemotherapy, ${ }^{12}$ indicating the importance of a multidisciplinary approach both at diagnosis and throughout the cycle of care such that the patient's oncologic treatment is always appropriately prioritized.

Finally, further investigation is needed to address factors that might affect omission of PMRT but were not evaluated by Miniani et al. ${ }^{8}$ One NCDB-specific limitation is the 
absence of data on human epidermal growth factor receptor 2 (HER2)/neu amplification/overexpression and the lack of distinction between use of hormonal therapy and chemotherapy with or without targeted therapy. The use of systemic therapy and how this influences the type of axillary surgery performed is an important area that is evolving and may not have been fully realized for patients treated during the period of the current study. As systemic therapy is increasingly administered in the neoadjuvant setting, ${ }^{13}$ consensus regarding the use of PMRT for patients undergoing upfront surgery versus those receiving neoadjuvant systemic therapy will be important as the field evolves. Use of PMRT is an important quality measure that also has the benefit of being relatively easy to measure and thus may prove to be a good surrogate for the quality of breast cancer care delivered at hospitals throughout the country.

Currently, the $\mathrm{CoC}$ requires that $90 \%$ of breast cancer patients with four or more positive lymph nodes receive PMRT within 1 year after diagnosis for an institution to be deemed compliant with the CoC's quality of care measure for PMRT. ${ }^{9}$ Expanding this benchmark to include recommended compliance levels for a broader set of indications for PMRT could further improve compliance not only with standards for radiotherapy but also with other measures of high-quality breast cancer care.

\section{REFERENCES}

1. Early Breast Cancer Trialists' Collaborative. Effect of radiotherapy after breast-conserving surgery on 10-year recurrence and 15-year breast cancer death: meta-analysis of individual patient data for 10,801 women in 17 randomised trials. Lancet. 2011;378:1707-16.

2. Jagsi R, Abrahamse P, Morrow M, et al. Patterns and correlates of adjuvant radiotherapy receipt after lumpectomy and after mastectomy for breast cancer. J Clin Oncol. 2010;28:2396-403.
3. Bilimoria KY, Bentrem DJ, Stewart AK, et al. Lymph node evaluation as a colon cancer quality measure: a national hospital report card. JNCI J Natl Cancer Institute. 2008;100:1310-7.

4. Kanemitsu Y, Komori K, Ishiguro S, et al. The relationship of lymph node evaluation and colorectal cancer survival after curative resection: a multi-institutional study. Ann Surg Oncol. 2012;19:2169-77.

5. Chen SL, Steele SR, Eberhardt J, et al. Lymph node ratio as a quality and prognostic indicator in stage III colon cancer. Ann Surg. 2011;253:82-7.

6. Senthil M, Trisal V, Paz I, et al. Prediction of the adequacy of lymph node retrieval in colon cancer by hospital type. Arch Surg. 2010;145:840-3.

7. Mathis KL, Green EM, Sargent DJ, et al. Surgical quality surrogates do not predict colon cancer survival in the setting of technical credentialing: a report from the prospective COST Trial. Ann Surg. 2013;257:102-7.

8. Minami C, Bilimoria K, Hansen N, et al. National evaluation of the new commission on cancer quality measure for post-mastectomy radiation treatment for breast cancer. Ann Surg Oncol. 2016. doi:10.1245/s10434-016-5257-5.

9. American College of Surgeons. CoC Measures for Quality of Cancer Care. Accessed 12 Feb 2016. https://www.facs.org/ quality\%20programs/cancer/ncdb/qualitymeasures.

10. Gradishar WJ, Anderson BO, Balassanian R, et al. Breast Cancer Version 1.2016. J Natl Comprehensive Cancer Network. 2015;13:1475-85.

11. American College of Surgeons. CoC Accreditation Categories. Accessed 12 Feb 2016. https://www.facs.org/quality-programs/ cancer/accredited/about/categories.

12. Haffty BG, McCall LM, Ballman KV, et al. Radiation use and field design in women with node-positive breast cancer treated with preoperative systemic therapy and breast surgery including axillary dissection on a prospective clinical trial ACOSOG Z1071 [Alliance]. Int J Radiat Oncol Biol Phys. 2015;93:S136.

13. Killelea BK, Yang VQ, Mougalian S, et al. Neoadjuvant chemotherapy for breast cancer increases the rate of breast conservation: results from the National Cancer Database. J Am Coll Surg. 2015;220:1063-9. 